\title{
Allocation of visual attention in younger and older adults
}

\author{
ALAN A. HARTLEY \\ Scripps College, Claremont, California \\ JAMES KIELEY \\ Pitzer College, Claremont, California \\ and \\ CRAIG R. M. MCKENZIE \\ University of Chicago, Chicago, Illinois
}

\begin{abstract}
Younger and older adults were compared in three experiments, using procedures that had been shown to affect the spread of visual attention. The attentional effects found in previous experiments were replicated. A broader focus of attention speeded responses to peripheral targets. In addition, two established findings concerning aging were replicated: Responses were slower in older than in younger adults, and, in certain conditions, they slowed more rapidly as target eccentricity increased. No interactions of age effects with attentional manipulations were found. The results of all three experiments were consistent with the interpretation that younger and older adults do not differ in the allocation of attention.
\end{abstract}

Information about the spatial location in which a stimulus will occur can facilitate the processing of that stimulus. The information can be an instruction (as in Hoyer \& Familant, 1987), a symbolic cue (as in Nissen \& Corkin, 1985), or a prior stimulus appearing in the same or a nearby location (as in Shulman, Wilson, \& Sheehy, 1985). The facilitation is not due to a shift in gaze, because it occurs even when the advance information precedes the stimulus by less than the time required for a saccadic eye movement (Posner, 1980). Instead, the facilitation can be attributed to a shift in attention. Attention is of interest in studies of age-related differences in cognitive function, because changes with advancing age in a basic process such as attention could provide a parsimonious explanation for the widespread differences that are seen in higher level performance. It has been specu-

We gratefully acknowledge support for this research from National Institute on Aging Grant AG01073, Claremont Graduate School, the Social Sciences Research Laboratory at the University of California, Irvine, and Scripps College Faculty Research Funds. Support for the first author during the preparation of this report was provided by $\mathrm{Na}$ tional Institute on Aging Senior Postdoctoral Fellowship F33 AG05433 and the School of Psychology at Georgia Institute of Technology. We are indebted to Vincent Brown for working out the procedure for obtaining maximum likelihood estimates of the reaction time-eccentricity slopes. We are grateful to Heidi Cover, Minh Thu Le, and Kirk Reynolds for assistance in data collection, and to Mary Duncan, Myron Braunstein, and Michael Butler for material support. Preliminary reports of portions of this work were presented at the Psychonomic Society meetings in Boston, MA, November 1986, and in Seattle, WA, November 1987, and at a conference on The Aging of Attention at the National Institute on Aging, November 1987. Correspondence concerning this article may be directed to Alan Hartley, Scripps College, Claremont, CA 91711 (e-mail: ahartley@pitvax.claremont.edu). lated that older adults may suffer from deficits in attention, in comparison with younger adults (Hoyer \& Plude, 1982). If attention is thought of as a resource that can be brought to bear on the processing of a stimulus, deficits could result if older adults allocated their resources less effectively than younger adults, if they had fewer resources, or both. In the experiments reported here, the allocation of attention in younger and older adults was explored in order to evaluate those alternative possibilities.

There is evidence that the focus of attention can be spread or narrowed. Some of this evidence is based on the examination of the costs and benefits of correct or incorrect advance cues about the location in which a target will appear on a trial (see, e.g., Egly \& Homa, 1984; Eriksen \& St. James, 1986; Eriksen \& Yeh, 1985; Jonides, 1980; Juola, Crouch, \& Cocklin, 1987).

LaBerge (1983) used a different approach. He attempted to control the focus of attention not by giving cues on each trial, but by manipulating the demands of the task. In a narrow-focus condition, the task required a judgment about one letter within a word or a nonword anagram of a word. In a wide-focus condition, the task required a judgment about an entire word. In one version of the procedure, there were occasional probe trials on which a target was presented at one of the positions where letters appeared on the word or nonword trials. The assumption was that the allocation of attention would be controlled by the primary task: Attention should be constricted to the size of a single letter in the narrow-focus conditions and broadened to the size of a word in the wide-focus conditions. Latencies of responses to the probe stimuli were assumed to provide a snapshot of the allocation of attention. Results confirmed the assumption. In the narrow- 
focus conditions, latencies increased as the target was farther from the attended letter location. In the wide-focus conditions, latencies were independent of position; the reaction time-probe position function was flat. These results are consistent with the view that attention can be spread broadly when that is advantageous for task performance.

Studies of aging and visual attention have been largely concerned with cuing (e.g., Hartley, Kieley, \& Slabach, 1990; Hoyer \& Familant, 1987; Nissen \& Corkin, 1985) and with search (e.g., Madden, 1982, 1983, 1984; Plude \& Doussard-Roosevelt, 1989; Plude \& Hoyer, 1986; Plude et al., 1983). When advance cues to the location of a target are given, the benefits of a correct cue and the costs of an incorrect cue are the same or larger for older adults in comparison with younger adults, although Hartley et al. (1990) argue that this may be accounted for by generally slower processing in the older adults. In studies of search, the individual must determine whether or not a multi-item display contains a target from a prespecified set. The time to respond increases as the number of elements in the display increases, and the increase is greater for older than for younger adults. Again, it has been argued that the age differences may be due to generalized slowing (Plude \& Doussard-Roosevelt, 1989). The display-size effect can be substantially reduced if the targets are always drawn from the same set (termed consistent mapping). Consistent mapping reduces the displaysize effect more for younger adults than for older adults. With extensive training, the reaction time (RT) becomes largely independent of the display size for younger adults but not for older adults (Fisk, McGee, \& Giambra, 1988). In summary, age differences have been found in attentional tasks, but they may have been the result of slowed processing in the older adults, rather than the result of deficits in attention.

In these prior studies, researchers have looked for age differences in the way in which attention is redeployed in response to a cue or in the way in which it is deployed when a multielement display must be searched. Such studies do not tell us whether there are age differences in the way in which attention is allocated across visual space when a stimulus of a particular size is expected in a particular location, and when there is ample time to prepare. Are older and younger adults equally able to narrow the focus of their attention? Are they equally able to broaden it? LaBerge's (1983) paradigm provides procedures that can be used to answer these questions. Those procedures were used in the first experiment reported here.

\section{EXPERIMENT 1}

The first experiment was a straightforward replication of the procedure used by LaBerge (1983, Experiment 1), extended to compare younger and older adults. A narrow focus of attention was induced by instructions to base the response on the third letter of a five-letter word or a nonword anagram of a word. A broad focus was induced by instructions to base the response on the entire word.

\section{Method}

Participants. The older adults in the experiments reported here were volunteers recruited at the Joslyn Senior Center in Claremont, $\mathrm{CA}$, as well as others referred by those individuals. The younger adults were students from the Claremont Colleges who volunteered to participate and students from social science courses at the University of California, Irvine, who participated in return for course credit. The characteristics of the participants in each of the experiments are shown in Table 1.

Display. Stimulus display and response recording were controlled by an Apple II + microcomputer. Timing was accomplished by independently calibrated software routines. The stimuli were displayed on a high-resolution Amdek monitor placed approximately $50 \mathrm{~cm}$ from the participants' eyes. Characters on the display subtended approximately $0.54^{\circ}$ horizontally and $0.80^{\circ}$ vertically. Characters were separated by approximately $0.15^{\circ}$. The sequence of events on each trial is shown in Table 2 . Each trial began with a warning signal, which consisted of a horizontal string of five \# characters displayed for $1,250 \mathrm{msec}$. The string was displayed in one of four positions across the horizontal midline of the display screen. The instructions were to fix one's gaze on the third character of the warning signal and to maintain it there throughout the trial. The stimulus following the warning signal was a horizontal string of five characters, which appeared in the same locations as did the characters of the warning signal. The strings of five characters subtended approximately $3.30^{\circ}$ (five characters at $.54^{\circ}$ and four spaces at $\left..15^{\circ}\right)$. They were displayed until a response was given or until 1,500

Table 1 Characteristics of Participants

\begin{tabular}{|c|c|c|c|c|c|c|}
\hline Experiment & No. Women & No. Men & $\begin{array}{c}\text { Mean Age } \\
\text { (Years) }\end{array}$ & $\begin{array}{c}\text { Education } \\
\text { (Years) }\end{array}$ & $\begin{array}{l}\text { Rated } \\
\text { Health }\end{array}$ & $\begin{array}{l}\text { Median } \\
\text { Acuity }\end{array}$ \\
\hline \multicolumn{7}{|c|}{ Younger Adults } \\
\hline 1 & 8 & 3 & 26.2 & 17.5 & 8.7 & \\
\hline 2 & 20 & 8 & 21.3 & 14.3 & 8.6 & $20 / 20$ \\
\hline 3 & 25 & 12 & 22.2 & 14.5 & 8.8 & $20 / 20$ \\
\hline \multicolumn{7}{|c|}{ Older Adults } \\
\hline 1 & 8 & 3 & 70.9 & 13.6 & 8.9 & \\
\hline 2 & 19 & 8 & 72.9 & 14.7 & 9.0 & $20 / 25$ \\
\hline 3 & 17 & 9 & 73.5 & 17.4 & 9.1 & $20 / 25$ \\
\hline
\end{tabular}

Note-Health was rated on a 10-point scale, with 10 as excellent. Acuity was measured using the Rosenbaum Pocket Vision test at a distance of $36.8 \mathrm{~cm}$ (14.5 in.). Participants in Experiment 1 were screened for their ability to read the display, but their acuity was not measured. 
Table 2

Events on Each Trial in Experiment 1

\begin{tabular}{lccc}
\hline & \multicolumn{3}{c}{ Condition } \\
\cline { 2 - 4 } & Word & Letter-Word & Letter-Nonword \\
\hline Warning Signal & \#\#\#\#\# & $\# \# \# \#$ & $\# \# \# \#$ \\
Primary Stimulus & & & \\
Positive set & ALICE & CABLE & ACBLE \\
$\quad$ Negative set & CHAIR & PORCH & OPRHC \\
Probe Stimulus & & & \\
Positive Set & $+++7+$ & $7++++$ & $+7+++$ \\
Negative Set & $\mathrm{Z}++++$ & $+++\mathrm{T}+$ & $++\mathrm{Z}++$ \\
\hline
\end{tabular}

msec had elapsed. The intertrial interval was $750 \mathrm{msec}$. The participant responded to stimuli in the positive set by pressing the space bar on the keyboard of the microcomputer. When stimuli were elements of the negative set, the participant was to withhold a response.

Stimuli and trial types. Trials included both primary and probe trials. There were three conditions that differed in the primary stimuli. In the word condition, the positive set was made up of fiveletter common first names (e.g., NANCY or STEVE); the negative set was made up of five-letter common nouns from the categories of furniture, musical instruments, and dwellings (e.g., CHAIR or PIANO). In the letter-word condition, the primary stimuli were familiar five-letter words with the middle (third) letter falling in the alphabet between $A$ and $G$, inclusively, in the positive set, and between $\mathrm{N}$ and $\mathrm{U}$ in the negative set. The stimuli in the letter-nonword condition were the same as those in the letter-word condition, except that the first, second, fourth, and fifth letters had been rearranged to form a nonword of low pronounceability (e.g., HRACI or NPAOI). The probe trials in all conditions consisted of four + signs and one critical, target item, 7, Z, or T. Across trials, the target item appeared at all five of the positions in the display string. On the probe trials, stimuli containing a 7 were in the positive set and those containing a $\mathrm{Z}$ or $\mathrm{T}$ were in the negative set. The critical items could appear at fixation $\left(0^{\circ}\right)$ or at $0.69^{\circ}$ or $1.38^{\circ}$ to the left or right of fixation. On each trial, either a primary or a probe stimulus appeared after the warning signal.

Procedure. The procedure was identical to that used by LaBerge (1983). Each participant completed one practice and two test blocks in each of the three primary task conditions. The order of conditions was chosen randomly, with the restriction that no order could be repeated until all orders had been used. The experiment began with an initial practice block that included only probe trials; there were 25 trials, with the 7 appearing three times at each of the five positions and $\mathrm{Z}$ or $\mathrm{T}$ appearing twice. Each condition began with a practice block of 50 trials, comprising 20 positive-set trials from the primary stimuli for that condition, 10 negative-set primary trials, and 15 positive- and five negative-set probe trials. There were two 80-trial test blocks in each condition: 45 positive- and 15 negativeset primary trials; 15 positive-set trials with the 7 appearing three times at each of the five positions; and five negative-set trials with $\mathrm{Z}$ or $\mathrm{T}$ appearing once at each position. Misses and false alarms were signaled by an error message throughout the experiment. To emphasize the primary task, participants were given feedback about average RT and errors for primary stimuli at the end of each practice and test block. They were encouraged by the instructions on the display screen to improve their RTs while maintaining their accuracy.

\section{Results}

The initial dependent variables for analysis were the mean RTs to the primary stimuli and to probes for each position in which the target item could appear. RTs shorter than $200 \mathrm{msec}$ or more than $2 S D$ s above the participant's average in that condition were discarded as outliers in computing the means.

For analysis of the variance in primary task RTs, age group (older or younger) was a between-subject factor, and condition (word, letter-word, and letter-nonword) was a within-subject factor. In this and subsequent analyses, the level of significance of a result is $p<.001$, unless stated otherwise. The analysis of variance showed significant effects of age group $[F(1,20)=46.53]$ and condition $[F(2,40)=8.15]$. Older adults $(M=655 \mathrm{msec})$ were slower than younger adults $(M=506 \mathrm{msec})$. The word condition produced shorter RTs $(M=550 \mathrm{msec})$ than did the letter-word $(M=604 \mathrm{msec})$ or the letternonword $(M=586 \mathrm{msec})$ condition.

For analysis of the variance in probe task RTs, age group (older or younger) was a between-subject factor, and condition (word, letter-word, and letter-nonword) and target location (center and inner and outer on the left and right) were within-subject factors. The average RTs are shown in Figure 1. The analysis of the variance showed significant effects of age group $[F(1,20)=19.04]$ and probe location $[F(4,80)=12.51]$, as well as significant interactions of age and location $[F(4,80)=2.57$, $p<.05]$ and condition and location $[F(8,160)=3.42]$. Examination of Figure 1 shows that older adults were slower than younger adults. RTs generally slowed with increasing distance of the critical item in the probe from fixation. This was qualified by a greater increase for older

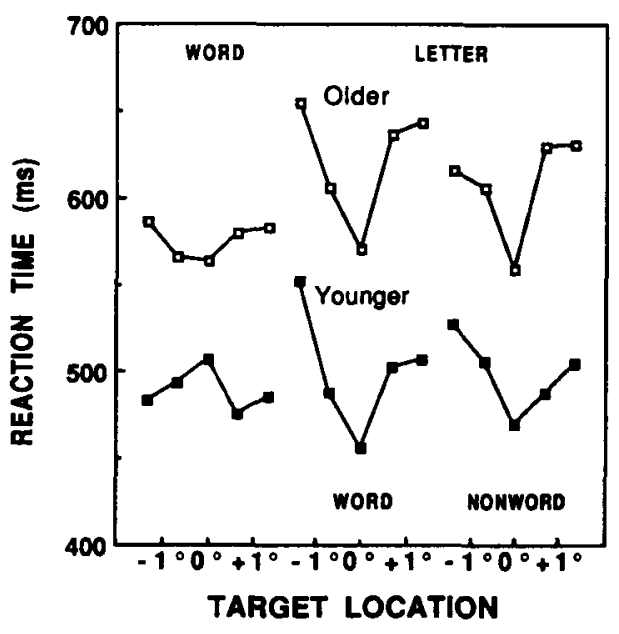

Figure 1. Reaction time to probes in Experiment 1, as a function of probe location and primary task (Word-names were discriminated from common nouns; Letter-Word-words with center letter a-g were discriminated from those with center letter $\mathrm{m}-\mathrm{u}$; Letter-Nonword-anagrams with center letter a-g were discriminated from those with center letter $m-u$ ). Filled symbols represent younger adults; open symbols represent older adults. 
Table 3

Maximum Likelihood Estimate Slopes of RT-Eccentricity Functions (in Milliseconds/Degree) as a Function of Age Group and Condition in Experiments 1 and 3

\begin{tabular}{lcc}
\hline & \multicolumn{2}{c}{ Age Group } \\
\cline { 2 - 2 } Condition & Younger & Older \\
\hline & Experiment 1 & \\
Word & -14.1 & 15.2 \\
Letter-word & 38.5 & 39.4 \\
Letter-nonword & 24.8 & 30.0 \\
& Experiment 3 & \\
Word & & \\
Narrow & 10.4 & 10.8 \\
Medium & 3.6 & 13.3 \\
Wide & 12.7 & 16.2 \\
Letter & & \\
$\quad$ Narrow & 33.4 & 56.8 \\
Medium & 30.8 & 32.5 \\
Wide & 23.2 & 32.8 \\
\hline
\end{tabular}

adults than for younger adults and a greater increase in letter-focus conditions than in the word-focus condition.

The change in RT with increasing target eccentricity can be captured by fitting a straight line separately to the left and right limbs of the RT function in each condition. Although there is some suggestion of nonlinearity, the slope of the best-fitting line provides a convenient summary of the increasing cost as stimuli deviate from fixation without doing substantial injustice to the data (see also Cerella, 1985; LaBerge \& Brown, 1986, 1989). Unfortunately, ordinary least squares slopes fit to the three points on a limb make use of information from only two of the points. LaBerge and Brown (1986) provide an alternative procedure, but that requires fixing the intercept of the function at the RT for the center target. The approach that was used was to calculate maximum likelihood estimates of the slopes for each limb, using the information from all five points. Analysis of variance of the slopes had age group as a between-subject factor and condition and side (left and right) as within-subject factors. The average slopes are given in Table 3 . There was a significant effect of condition $[F(2,40)=11.58]$, and there was a significant interaction of age and side $[F(1,20)=$ $5.83, p<.05$ ]. The effect of age was not significant $[F(1,20)=2.00, p=.17]$. Slopes in the letter-word and letter-nonword conditions $(M \mathrm{~s}=39.0 \mathrm{msec} / \mathrm{deg}$ and $27.4 \mathrm{msec} / \mathrm{deg}$ ) were higher than in the word condition $(M=0.6 \mathrm{msec} / \mathrm{deg})$. Word slopes did not differ significantly from zero [older adults, $t(10)=.96$; younger adults, $t(10)=-.94 \mathrm{~J}$. Older adults had slightly steeper slopes for targets to the right, while younger adults had steeper slopes for targets to the left.

Errors occurred on $2.5 \%$ of the probe trials for older adults and on $3.2 \%$ of the probe trials for younger adults. Analysis of the variance in errors showed no significant effects.

\section{Discussion}

The results replicate LaBerge's (1983) findings and confirm his conclusion that the focus of spatial attention is broader when it is directed to a word than when it is directed to a single letter. When attention was focused on a single letter, RTs were fastest when the target appeared at the same position as the letter did. RTs increased as the target was farther from the focus. This was not true when attention was focused on the entire word; RTs were independent of target location. Although older adults were slower overall, the effects of the experimental manipulation of focus size did not interact with age. This result is consistent with the interpretation that the allocation of attention is the same in younger and older adults.

Attention might be thought of as a spotlight (e.g., Eriksen \& Yeh, 1985; LaBerge, 1983; Posner, 1978; Remington \& Pierce, 1984; Shulman, Remington, \& McLean, 1979; Tsal, 1983). If the spotlight were narrowly focused on the central location of a five-letter string, a target at that location would require no movement of attention. If the target appeared elsewhere, the attentional spotlight would have to be redirected. If movement of the spotlight were analog, RTs would increase monotonically with increasing distance of the target from the original focus of attention. The RTs in the letter-focus conditions resemble such a V-shaped function. The flat RT functions in the word-focus condition imply that it must be possible to broaden the spotlight to encompass five-letter words. If the focus could not be broadened beyond a single letter, the best strategy would be to leave the focus on the center letter, because that would minimize the average distance to a target. Such a strategy would have produced similar results in the word and letter conditions. The results also could not be accounted for by assuming that the focus was one letter in width and that it was trained at random on one of the five target locations. As LaBerge (1983) has shown, this would produce a U-shaped RT function with substantially slower reactions to the most eccentric targets than to more central targets.

Attention might also be conceptualized as a distribution of processing resources across the visual field (Downing, 1988; Downing \& Pinker, 1985; Jonides, 1980; LaBerge \& Brown, 1989). The speed with which a stimulus is processed would thus be a function of the resources allocated to the spatial location in which it appears. In this view, the distribution would be peaked at the location of the central letter in the letter conditions, and it would fall away rapidly with increasing eccentricity. Targets appearing at the location of the letter would be responded to most quickly, because that location has the most resources. RTs to probes at other locations would increase with eccentricity as the height of the distribution at those locations diminished. In the word condition, resources would be spread equally across the five locations in which the target might appear, so RTs would be independent of target position.

To emphasize, the major finding was that the effects of target eccentricity were different when attention had previously been directed to a word than when attention had been focused on a single letter. Because the targets were identical in the two conditions, the perceptual quality of the target was the same. The difference must have 
Table 4

Events on Each Trial in Experiment 2

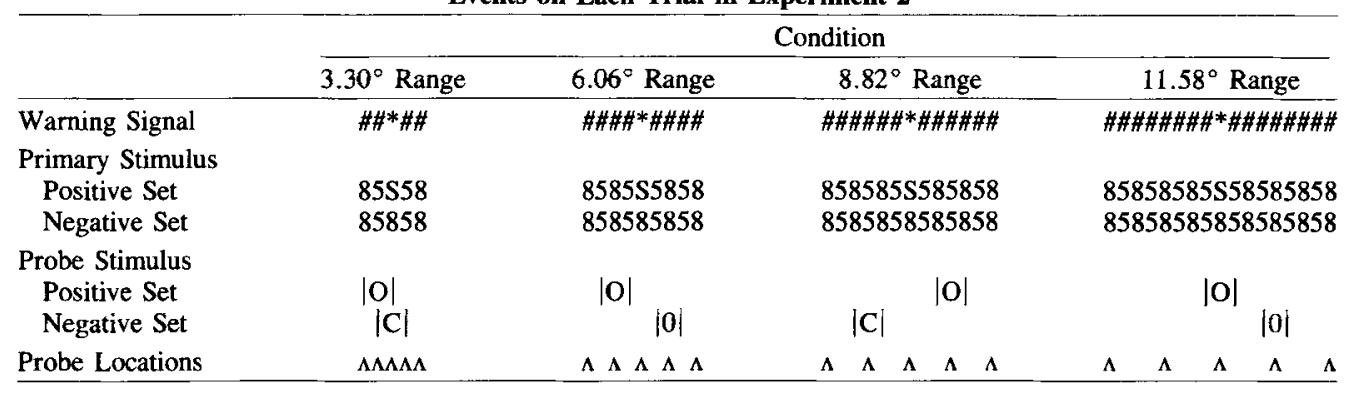

been due to the different allocation of attention. The difference between word-focus and letter-focus conditions was unaffected by age. So there was an attentional effect, and it was independent of age.

Whichever metaphor is adopted for the spread or narrowing of attention, older and younger adults appear to respond to the demands of the situation. They are equally able to broaden the attentional spotlight or to spread attentional resources.

\section{EXPERIMENT 2}

Using another procedure, LaBerge and Brown (1986) have reported results that appear to reflect the spatial distribution of attention. They found that the V-shaped RT function that LaBerge (1983) had obtained in the letterfocus conditions was affected by the range over which the targets could occur. The procedure was a variant of that used by LaBerge (1983, Experiment 2). Instead of a probe's being presented only on occasional trials, both primary and probe stimuli appeared on every trial. The primary stimulus was a string of $8 \mathrm{~s}$ and $5 \mathrm{~s}$ with either an $S$ or an 8 at the center. The probe stimulus was $|O|$, $|C|$, or $|0|$. The task was to respond if the primary stimulus contained an $\mathrm{S}$ and the probe contained an $\mathrm{O}$, and to withhold a response otherwise. The results were the same as those from the letter-focus task. RTs increased as the probe target was farther from the center of the primary stimulus. The principal manipulation was to vary the range over which probe targets could occur, from $1.7^{\circ}$ to $8.6^{\circ}$. As the range increased, the slopes of the RT-eccentricity functions became shallower. The most eccentric stimuli were responded to equally rapidly, regardless of absolute distance from fixation. LaBerge and Brown (1986) called this an attention-range effect. It was as though the deployment of attention was a function of the range of possible target locations. Nonetheless, the shape of the RT-eccentricity functions indicated that attention was still concentrated at fixation, presumably to deal with the primary stimulus. In Experiment 2, we adopted LaBerge and Brown's (1986) procedures to probe for age differences in the spread of attention.

\section{Method}

Display and trial types. The characteristics of the display were similar to those in Experiment 1 except that a high-resolution EA amber monitor (EU-2131N) was used. To maintain a constant distance from the display, the participant was asked to keep his or her forehead in contact with a strip fixed at $36.8 \mathrm{~cm}$ (14.5 in.) from the screen. The events on each trial are shown in Table 4. Each trial began with a warning signal displayed for $1,000 \mathrm{msec}$. The warning signal was immediately followed by a primary stimulus, which remained on for $200 \mathrm{msec}$. This was a string of alternating $8 \mathrm{~s}$ and $5 \mathrm{~s}$ with either an $S$ or an 8 at the center. The primary stimulus was immediately followed by a probe stimulus-- $|\mathrm{O}|,|\mathrm{C}|$, or $|0|-$ which erased the primary stimulus and remained on for $200 \mathrm{msec}$. An additional 1,200 msec were allowed for a response. The task was to respond by pressing the space bar if the primary stimulus contained an $S$ at the center and the probe stimulus contained an $O$, and to withhold a response otherwise. The latency timer was started at the start of the probe display and stopped by the participant's keypress.

The four conditions differed in the range covered by the stimuli. In each condition, there were five target locations in which the probe stimuli could appear. In the narrowest range, the target character in the probe could appear centered at fixation $\left(0^{\circ}\right)$, or $.69^{\circ}$ or $1.38^{\circ}$ to the left or right of fixation. The total range of targets was approximately $3.30^{\circ}$ between the outer contours of characters at the far left and far right probe locations (five character spaces at $.69^{\circ}$ less $.15^{\circ}$ space bordering the outside characters). In the next condition, there was one character space between adjacent target positions. Targets appeared at $0^{\circ}$ or $1.38^{\circ}$ or $2.76^{\circ}$ to the left or right of fixation. The total range was $6.06^{\circ}$ (nine character positions at $.69^{\circ}$ less $.15^{\circ}$ ). In the next wider condition, there were two character spaces between adjacent positions. Targets appeared at $0^{\circ}$ or $2.07^{\circ}$ or $4.14^{\circ}$ to the left or right of fixation. The total range was $8.82^{\circ}$ $\left(13\right.$ character positions at $.69^{\circ}$ less $\left..15^{\circ}\right)$. In the widest condition, there were three character spaces between adjacent positions. Targets appeared at $0^{\circ}$ or $2.76^{\circ}$ or $5.52^{\circ}$ to the left or right of fixation. The total range was $11.58^{\circ}\left(17\right.$ character positions at $.69^{\circ}$ less $.15^{\circ}$ ). In each condition, the warning signal and the primary stimulus subtended the same extent as the range of possible targets, with a character in every position (i.e., warning signals were

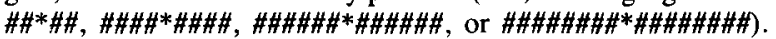

Procedure. Each subject completed a 40-trial practice block and four 80-trial test blocks. The practice block included examples of trials at all four ranges. Over the practice trials, the display times for both the primary and the probe stimuli were gradually reduced from 1,200 to $200 \mathrm{msec}$. Each test block contained trials at only one range. The order of test blocks was randomly determined. Each test block had 40 positive-set trials (i.e., $\mathbf{S}$ followed by $\mathrm{O}$ ), eight at each possible probe target location. In addition, there were 40 


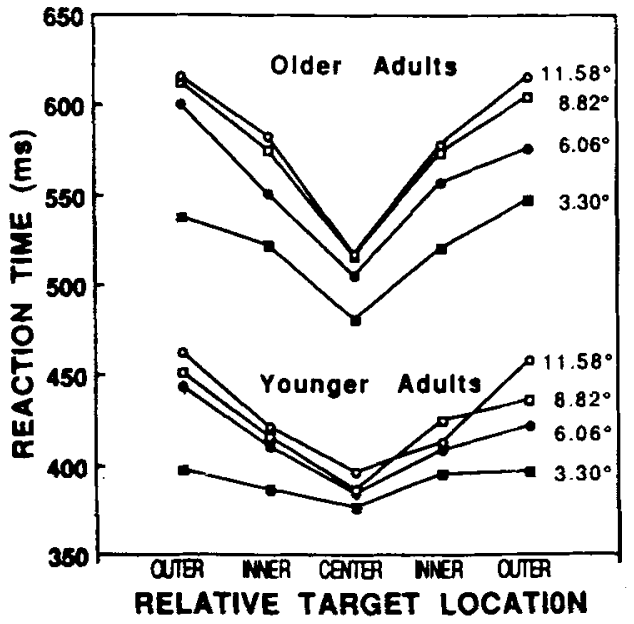

Figure 2. Reaction times to probes in Experiment 2, as a function of relative probe location, width of field of primary task, and age group.

negative-set trials, divided into 20 on which $S$ appeared but was not followed by $O$ and 20 on which $S$ did not occur. Feedback about average speed was given at the end of the practice block. The participant was encouraged to increase speed while maintaining accuracy. If there were more than five errors on the practice block, instructions on the display encouraged the participant to improve both speed and accuracy and allowed an opportunity to repeat the practice if desired.

\section{Results}

An analysis of variance in the mean RTs to the probe stimuli (after trimming outliers) had age group as a between-subject factor and probe target range $\left(3.30^{\circ}\right.$, $6.06^{\circ}, 8.82^{\circ}$, and $11.58^{\circ}$ ) and probe target location (left outer, left inner, center, right inner, right outer) as withinsubject factors. The analysis produced significant main effects of age group $[F(1,53)=33.71]$, target range $[F(3,159)=36.99]$, and target location $[F(4,212)=$ 104.67]. There were significant interactions of age and location $[F(4,212)=7.98]$ and range and location $[F(12,636)=4.54]$. Examination of the means, given in Figure 2, shows that the older adults were slower than the younger adults. RTs increased with target range and with target eccentricity. The effect of eccentricity was larger for the older adults than for the younger adults, and it also increased with increasing range. An analysis of variance of RTs for the center target position only showed that older adults were slower $[F(1,53)=23.11]$. There was also an effect of range $[F(3,159)=5.77]$. The two variables (age, range) did not interact $[F(3,159)=$ $1.11, p=.34]$. Average center-position RTs increased monotonically from the narrowest range $(429 \mathrm{msec})$ to the widest $(457 \mathrm{msec})$.

An analysis of variance in the slopes of the best-fitting lines produced significant effects of age group $[F(1,53)=$ $23.73]$ and range $[F(3,159)=8.58]$, as well as a significant interaction of age and range $[F(3,159)=6.74]$. Slopes were steeper for older adults $(M=38.0 \mathrm{msec} / \mathrm{deg}$ ) than for younger adults $(M=19.7 \mathrm{msec} / \mathrm{deg})$. Generally, slopes declined as probe target range increased, as can be seen in Table 5, except for the very shallow slope for younger adults in the narrowest field, which was responsible for the interaction of age and range.

LaBerge and Brown (1986) found that a straight line fit to RTs from the outer, most eccentric target locations had zero slope, indicating that RTs were independent of target range. The same was true for a line fit to the inner, noncenter points. Similar analyses of the present data produced somewhat different results, which are given in Table 6. In every case, the slopes did differ from zero, indicating that RTs to the same relative position slowed as the absolute distance of that position from fixation increased.

Analysis of the variance in the percentage of errors showed significant effects of age group $[F(1,41)=25.35]$, range $[F(3,123)=9.86]$, and location $[F(4,164)=$ 18.58]. (The degrees of freedom differ from those in the preceding analyses because error data for some of the participants were lost.) The older adults had a higher percentage of errors $(M=11.2)$ than did the younger adults $(M=3.7)$. Errors were most frequent in the $8.82^{\circ}$ and $11.58^{\circ}$ ranges $(M=10.0$ and 9.6$)$, intermediate for the $6.06^{\circ}$ range $(M=7.7)$, and lowest for the $3.30^{\circ}$ range $(M=5.4)$. Errors were higher for the outer positions ( $M=4.7$ for left, 4.8 for right) than for the inner positions ( $M=3.7$ for left, 3.1 for center, and 2.2 for right).

\section{Discussion}

There are two general questions to be answered. Was LaBerge and Brown's (1986) attention-range effect replicated? Does the same effect occur for both younger and older adults?

Table 5

Maximum Likelihood Estimate Slopes of RT-Eccentricity Functions (in Milliseconds/Degree) as a Function of Age and Target Range in Experiment 2

\begin{tabular}{lrrrr} 
& \multicolumn{4}{c}{ Target Range } \\
\cline { 2 - 5 } Age Group & $3.30^{\circ}$ & $6.06^{\circ}$ & $8.82^{\circ}$ & $11.58^{\circ}$ \\
\hline Younger & 18.9 & 24.5 & 18.6 & 16.9 \\
Older & 58.4 & 40.3 & 29.9 & 23.5 \\
\hline
\end{tabular}

Table 6

Tests on Slopes of Least Squares Best-Fit Lines Through Outer and Inner Target Locations as a Function of Target Range

\begin{tabular}{|c|c|c|c|c|}
\hline & \multicolumn{4}{|c|}{ Target Locations } \\
\hline & \multicolumn{2}{|c|}{ Inner } & \multicolumn{2}{|c|}{ Outer } \\
\hline & Left & Right & Left & Right \\
\hline \multicolumn{5}{|c|}{ Younger } \\
\hline Slope & 8.00 & 7.91 & 12.97 & 13.94 \\
\hline$t$ & $3.57+$ & $2.94 \ddagger$ & $9.37^{*}$ & $6.74^{*}$ \\
\hline \multicolumn{5}{|c|}{ Older } \\
\hline $\begin{array}{l}\text { Slope } \\
t\end{array}$ & $\begin{array}{c}15.68 \\
4.52^{*}\end{array}$ & $\begin{array}{c}11.71 \\
2.20 \ddagger\end{array}$ & $\begin{array}{c}14.33 \\
3.99 \dagger\end{array}$ & $\begin{array}{c}14.96 \\
3.50 \dagger\end{array}$ \\
\hline
\end{tabular}

Note-l tests are tests of the hypothesis that slopes are significantly different from zero. Degrees of freedom are 11 for each test. ${ }^{*} p<$ $.001 . \quad t p<.01 . \quad \$ p<.05$. 
Attention range effect. Consistent with LaBerge and Brown's (1986) results, the slopes of the RT functions decreased as the range increased. LaBerge and Brown found that the RTs to the outer points did not differ significantly; a straight line fit through them had zero slope. The same was true for the inner points. Those results were not found here. RTs increased as eccentricity increased. LaBerge (personal communication, March 2, 1987) has suggested that the difference in results may be due to a difference in procedure. The subjects in LaBerge and Brown's experiment completed each range condition on a different day. Those in the present study completed all conditions on the same day. This could have allowed carry-over effects of one field size into another. To test this possibility, a reduced version of the experiment with only the $6.06^{\circ}$ and $11.58^{\circ}$ ranges was repeated twice with younger adults, once as a between-subject design and once as a within-subject design. The results were comparable to those of LaBerge and Brown with a between-subject design and comparable to the results of the present experiment with a within-subject design: RTs to the most extreme positions did not vary as a function of range in the between-subject design; they increased with increasing eccentricity in the within-subject design.

Younger versus older adults. The performance of the older adults yielded steeper slopes than did that of the younger adults, but the older adults showed the attentionrange effect at least as clearly. In fact, the major deviation from the results of LaBerge and Brown (1986) was the unusually flat RT function for the younger adults in the narrowest range condition. It is not clear why this occurred. It is as though the younger adults spread their attention evenly over the field in this condition. LaBerge and Brown did not find such a result, although their young adults were from exactly the same pool at the University of California, Irvine, as were those in the present study. The performance of the young adults in the narrowest condition is perhaps best treated as an anomaly. The important result for the present purposes is that a manipulation presumed to affect the spread of attention had the same effect in older and in younger adults.

Theoretical interpretation. As LaBerge and Brown (1986) have pointed out, it is difficult to reconcile the attention range effect with either a spotlight or a resourcedistribution explanation of attention. The speed with which a spotlight moved would have to increase as the range of possible target locations increased. A resource explanation would require that additional resources be mobilized and allocated to peripheral positions as the range increased.

One possibility is that resources are allocated only to positions where targets can appear, regardless of the range over which those positions are spread. LaBerge and Brown (1986) showed that this was not the case. They found virtually identical functions, whether there were five or nine target locations within a particular range. We replicated their procedures with 10 younger and 10 older adults. RTs were slightly slower with nine target locations $(M=531 \mathrm{msec})$ than with five $(M=522 \mathrm{msec})$, but the slopes of the RT-eccentricity functions did not differ between nine locations $(10.6 \mathrm{msec} / \mathrm{deg}$ for the younger adults; $12.7 \mathrm{msec} / \mathrm{deg}$ for the older adults) and five locations (11.2 msec/deg for the younger adults; $17.1 \mathrm{msec} / \mathrm{deg}$ for the older adults).

RTs to the center target location increased as range increased. Thus it is possible that, as the range of target locations increased, resources were increasingly reallocated from the center position to the periphery. From the V-shaped RT functions, it is clear that attention remained focused primarily at fixation, presumably because that was the location of the critical character in the primary stimulus. That character not only required a difficult discrimination but occurred in a known location, so it seems reasonable that attention would have been directed to that location. It is possible, however, that some resources were directed away from that location as the range increased, and that processing of the primary stimulus suffered. The procedures used in Experiment 1 which provided RTs to both primary and probe stimuli would have allowed a direct examination of that possibility. This was done in Experiment 3.

\section{EXPERIMENT 3}

The stimuli in Experiment 1 covered a relatively small extent of the visual field. The stimuli in Experiment 2 covered a much broader extent, but it is not clear that the allocation of attention over the range of possible targets represents a deliberate broadening of attention. The allocation of attention that produces the attention-range effect must be secondary to the focusing of attention to process the primary stimulus. One possibility is that it is the result of an active process of allocating attention between the primary and secondary tasks. A second possibility, however, is that there are two processes, an active allocation of attention to the center of the field and a passive activation resulting from the occurrence of peripheral targets (cf. LaBerge \& Brown, 1989). One purpose of Experiment 3 , then, was to determine whether age differences would emerge as the focus of attention was actively widened over a broader extent of the visual field than in Experiment 1. To explore this possibility, the word condition from Experiment 1 was repeated, but two additional conditions were included in which the characters in the five-letter words were separated by one or two blank character spaces. Consequently, both the extent of the primary stimuli and the range of target positions in the probe stimuli extended approximately $3.30^{\circ}, 6.06^{\circ}$, or $8.82^{\circ}$.

A second purpose of Experiment 3 was to explore the attention-range effect further. Letter-word conditions were also included, to allow an additional examination of the effect. The primary task RTs should indicate whether attention is being redistributed from the center to the pe- 
riphery. The letter-nonword condition was omitted, because in Experiment 1, the results were very similar to those for the letter-word condition.

\section{Method}

Stimuli and trial types. The characteristics of the display were identical to those in Experiment 2. The word and letter-word conditions were each repeated with narrow, medium, and wide character strings. Narrow strings were identical to the stimuli of Experiment 1, with no character spaces between adjacent characters. Medium strings had one blank character space between characters in the warning signal, word, and probe strings. Targets on probe trials occurred centered at fixation $\left(0^{\circ}\right)$ and $1.38^{\circ}$ and $2.76^{\circ}$ to the left and right of fixation. The warning signal and primary stimuli subtended approximately $6.06^{\circ}$. Wide strings had two blank character spaces between adjacent characters. Targets on probe trials occurred at $0^{\circ}$ and $2.07^{\circ}$ and $4.14^{\circ}$ to the left and right. The warning signal and primary stimuli subtended approximately $8.82^{\circ}$. The stimuli for word and letter-word conditions were selected in the same manner as in Experiment 1. Primary stimuli were not repeated. The response rules were the same as in Experiment 1: for words, first names were the positive set and common objects were the negative set; for letters within words, A through $G$ were the positive set and $\mathrm{N}$ through $\mathrm{U}$ were the negative set. Unlike in Experiment 1, both the primary and probe stimuli were removed after $200 \mathrm{msec}$, to eliminate possible effects of eye movements. During the practice trials, the display duration was gradually reduced from 1,200 to 200 msec.

Procedure. Each participant completed 65-trial blocks of narrow, medium, and wide stimuli in both the word and letter-word conditions, for a total of 390 experimental trials. Half of the participants received all word trials first; the other half received all letter-word trials first. The order was alternated for successive participants within an age group. Trials within a condition were blocked by width, with the order of the three width conditions determined at random. A practice block of 40 trials preceded the experimental trials in each of the two focus conditions, word and letter-word. The practice block included trials from all three width conditions. In the experimental blocks, there were 35 positive-set, 10 negativeset, and 20 probe trials. The probe trials included 4 at each target location, 3 positive and 1 negative. The general instructions explained the task, emphasizing the primary task. The participants received feedback about both speed and accuracy after each practice block. They were encouraged to increase speed while maintaining accuracy. If there were more than five errors in the practice trials, the participant was offered the opportunity to repeat the practice block, and was encouraged to improve both speed and accuracy. Rest periods were given at the end of each experimental block.

\section{Results}

The word and letter-word conditions were analyzed separately.

Word conditions: Primary task. An analysis of variance was conducted on mean RTs (trimmed of outliers), with age group as a between-subject factor and field width (narrow, medium, and wide) as a within-subject factor. There were significant main effects of age group $[F(1,61)=$ $64.15]$ and field width $[F(2,122)=48.58]$, and a significant interaction of the two variables $[F(2,122)=3.76$, $p=.026]$. The means are shown in Figure 3. The older adults were slower than the younger adults. RTs increased with field width, more rapidly for the older than for the younger adults.

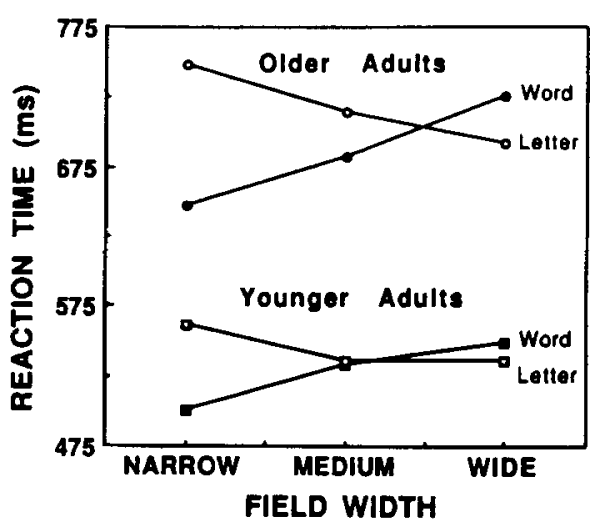

Figure 3. Reaction times in primary task in Experiment 3, as a function of width of field of primary task, type of primary task, and age group.

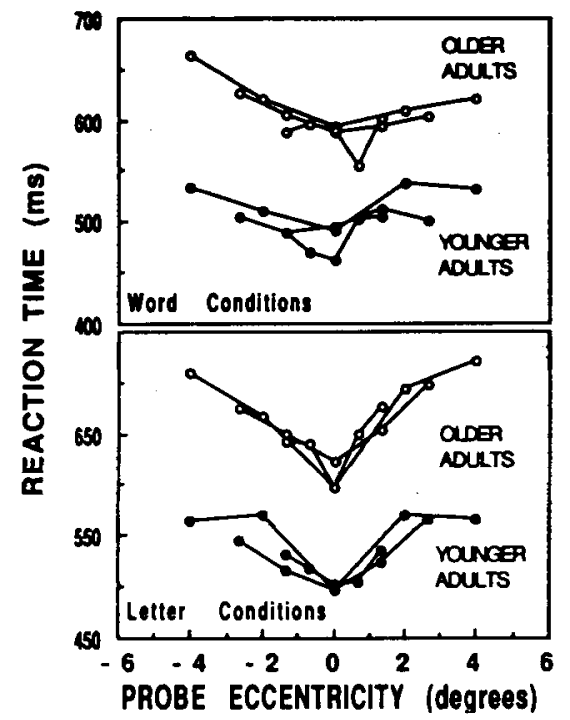

Figure 4. Reaction times to probes in Experiment 3, as a function of probe location, width of field of primary task, type of primary task, and age group.

Word conditions: Probe task RTs. For the analysis of mean RTs, age group was a between-subject factor and field width and target location (center and inner and outer on the left and right) were within-subject factors. There were significant main effects of age group $[F(1,61)=$ $40.26]$, field width $[F(2,122)=31.43]$, and target location $[F(4,244)=7.69]$. There were significant interactions of age group and target location $[F(4,244)=$ $4.65]$, and field width and target location $[F(8,488)=$ $2.62, p=.008$ ] . Examination of the means, shown in Figure 4, shows that the older adults were slower than the younger adults. Overall, RTs increased as field width increased (narrow, $M=522 \mathrm{msec}$; medium, $M=543 \mathrm{msec}$; wide, $M=562 \mathrm{msec}$ ). RTs also increased as target eccentricity increased, and the increase was greater with 
greater field width. A separate analysis was carried out on the center target position only. That analysis showed only that the older adults were slower than the younger adults $[F(1,61)=39.65]$. There was no effect of field width and no interaction of field width and focus condition. The interaction of age, focus, and field width was also nonsignificant $[F(8,488)=.70]$.

Word conditions: Probe task slopes. Interpretation of the RT results is simplified by looking at the slopes of the best-fitting lines fit to the RT-eccentricity function for each participant in each condition. The mean slopes are given in Table 3. Analysis of variance in the slopes showed no significant effects of age group $[F(1,61)=.06]$ or of field width $[F(2,122)=.45]$, nor was there a significant interaction $[F(2,122)=.74]$. The mean slope was $12.6 \mathrm{msec} / \mathrm{deg}$; this was significantly greater than zero $[t(61)=3.87]$. The absence of an effect of field width on the slopes combined with an interaction of width and target location in the RTs indicates that the RTs to peripheral positions became systematically longer as the position was in a more eccentric location. That is, the increase in RTs from fixation to the inner position to the outer position increased with target range, but the increase was proportionate to the change in the absolute location of those positions.

Word conditions: Errors. An analysis of variance in the error percentages showed no significant effects.

Letter conditions: Primary task. There were significant main effects of age group $[F(1,61)=43.42]$ and width $[F(2,122)=19.03]$. The means are shown in Figure 3. Older adults were slower. RTs decreased with increasing field width. There was a tendency for a greater decrease for older than for younger adults, but the effect was not significant $[F(2,122)=2.51, p=.086]$.

Letter conditions: Probe task RTs. There were significant main effects of age group $[F(1,61)=54.27]$, field width $[F(2,122)=16.04]$, and target location $[F(4,244)=$ 29.88]. There was a significant interaction of field width and target location $[F(8,488)=2.64, p=.008]$. The means are shown in Figure 4. The older adults were slower. Overall, RTs increased with field width (narrow, $M=569 \mathrm{msec}$; medium, $M=583 \mathrm{msec}$; wide, $M=$ $606 \mathrm{msec}$ ). RTs also increased as the location was more peripheral, and the increase was larger as the field width was greater. A separate analysis of RTs for the center location showed only a significant effect of age group $[F(1,61)=33.55]$

Letter conditions: Probe task slopes. There was a significant effect of the side [left vs. right; $F(1,61)=4.99$, $p=.029$ ]. The slope for the right limb ( $M=39.4 \mathrm{msec})$ deg) was greater than that for the left $\operatorname{limb}(M=$ $30.4 \mathrm{msec} / \mathrm{deg}$ ). Although the slopes decreased with increasing field width, as can be seen in Table 3, the effect was not significant $[F(2,122)=2.13, p=.123]$. The overall average slope was $34.9 \mathrm{msec} / \mathrm{deg}$, which was significantly greater than that for the word conditions $[t(61)=3.94]$.

Letter conditions: Errors. An analysis of variance in the error percentages showed no significant effects.

\section{Discussion}

The results from the word conditions demonstrated that older adults could broaden their attention as effectively as younger adults over a much wider range than that in Experiment 1 . The older adults were slower, but there was no significant difference in the slopes of the probe task RT-eccentricity functions for the two age groups. The slopes were significantly greater than zero, which was consistent with a progressive impairment in the processing of targets as they appeared farther in the periphery. In addition, the primary task RTs increased with field width. These two results could reflect either reduced visual acuity in the periphery or a difficulty in deploying attention over so broad an area. The important points are that the probe task slopes were significantly smaller than in the letter-focus conditions, indicating that attention was more broadly deployed in the word-focus conditions, and that the slopes did not differ for younger and older adults, indicating that attention was deployed as broadly in both groups.

In the letter-focus conditions, the slopes did decrease with an increasing range over which the probe targets could occur. The attention-range effect was not significant. It is likely that it was attenuated because the probe stimuli-requiring processing of targets away from primary task fixation-appeared only occasionally rather than on every trial, as in Experiment 2. Also, as in Experiment 2 , the use of a procedure in which subjects were exposed to all ranges in the same experimental session probably reduced the attention-range effect. Although the effect was not significant, the decrease in probe task RT slope with increasing range is consistent with the attentionrange effect observed in Experiment 2. The results also confirm the earlier finding that the attention-range effect is not different in younger and older adults.

The primary task RTs dropped with increasing field width in the letter conditions. The RTs to probes in the center location were unaffected by field width. These results are inconsistent with the explanation that the attentionrange effect is due to a reallocation away from fixation to the periphery. It is the case, however, that as the field width increased, the characters flanking the critical center character in the primary stimulus were farther away. This could have reduced interference sufficiently so that resources could be transferred to the periphery without impairing processing of the primary task or of probes at the center position. To explore this possibility, a small experiment was conducted with 12 younger adults. The stimuli and procedures were identical to those used in the narrow and medium letter-focus conditions of Experiment 3, except that the spaces between letters were filled with Xs (e.g., CXAXBXLXE). In this experiment, both the primary task RTs and the center-position probe RTs were lower for the medium condition than for the narrow condition (primary: narrow, $M=534 \mathrm{msec}$, medium, $M=$ $521 \mathrm{msec}, t(11)=.92$; probe: narrow, $M=444 \mathrm{msec}$, medium, $M=428 \mathrm{msec}, t(11)=1.13$ ]. So even when the amount of interference is equated across conditions, performance is better with the wider range. It appears, 
then, that the attention-range effect is not due to a reallocation of attention away from the center position. If a resource distribution explanation is to be used, the total amount of resources allocated to the task must increase as the range of possible targets increases. A spotlight explanation could explain the range effect, were one to suppose that the speed of movement of the spotlight increased as the range increased. No simple extension would explain the reduction in primary task RTs with increasing range.

\section{GENERAL DISCUSSION}

Manipulations that should have affected the spread of attention had effects, but they had the same effects in older as in younger adults. In Experiments 1 and 3, target eccentricity with a broad focus of attention affected RTs less than did target eccentricity with a narrow focus. In Experiment 2 and in the letter-focus condition of Experiment 3 with a narrow focus of attention, the slopes of the RT-eccentricity functions decreased as the range of possible target locations increased, which is consistent with an increasing spread of attention with a wider range over which targets could appear. In none of these experiments did the effect of the experimental manipulation interact with age. These results converge on the conclusion that the control of attention is unaffected by age.

It was true, however, that the slopes of the RT functions were consistently steeper for the older adults than for the younger adults across the three experiments, although the difference was not always significant. The weighted average slope for the older adults for Experiment 2 and for the letter-focus conditions of Experiments 1 and 3 was $38.5 \mathrm{msec} / \mathrm{deg}$; the slope for the younger adults was $26.1 \mathrm{msec} / \mathrm{deg}$. This result is consistent with an attentional spotlight that travels more slowly or with a distribution with lower total resources in older adults. If this were the case, however, the effects of age should have interacted with the effects of manipulations that would broaden the spotlight or change its speed or affect the allocation of resources. But the effects of age differences were independent of the effects of attentional manipulations. There is another explanation for the age differences-an impaired ability to process extrafoveal stimuli in the older adults. This could be due to a reduction in discriminability with increasing distance of the stimulus from the fovea of the eye, a reduction that is more pronounced in older adults. A perceptual effect such as this would not be expected to interact with attentional manipulations. However, in the word-focus conditions of Experiment 3 with attention presumably spread evenly across the field, the slopes for the younger ( $M=11.9 \mathrm{msec} / \mathrm{deg}$ ) and older $(M=13.4 \mathrm{msec} / \mathrm{deg})$ adults were very similar. This result is inconsistent with any differential impairment of perceptual processing of extrafoveal stimuli in older adults. The remaining explanation is strategic. It is possible that the older adults simply maintained a narrower focus of attention when the task required focusing on a single character.
These experiments replicated a number of findings previously attributed to attention and others attributed to aging. Although a variety of attentional manipulations were explored, they failed to show age differences in the static allocation of selective attention to portions of the visual field (with the one possible exception just noted). Although older adults are slower than younger adults, they do not appear to suffer from reduced attentional resources or ineffective allocation of those resources. The failure to find an interaction of age and an attentional manipulation in a single experiment might be dismissed as uninformative. The consistent absence of such interactions combined with the demonstration of established effects makes it more likely that younger and older adults do not differ in the spatial allocation of visual attention. This is an important finding on its own, but it is also important because age differences have been found in attention-requiring tasks such as visual search, which require rapid, repeated reallocations of attention within a trial. Findings of age similarities in some attention-demanding tasks and differences in others could help to identify the locus of age changes in cognitive functioning.

\section{REFERENCES}

Cerella, J. (1985). Age-related decline in extrafoveal letter penception. Journal of Gerontology, 40, 727-736.

Downing, C. J. (1988). Expectancy and visual-spatial attention: Effects on perceptual quality. Joumal of Experimental Psychology: Human Perception \& Performance, 14, 188-202.

Downing, C. J., Pinker, S. (1985). The spatial structure of visual attention. In M. I. Posner \& O. S. Marin (Eds.), Attention and performance XI: Mechanisms of attention (pp. 171-187). Hillsdale, NJ: Erlbaum.

EGLY, R., Homa, D. (1984). Sensitization of the visual field. Journal of Experimental Psychology: Human Perception \& Performance. 10, 778-793.

Eriksen, C. W., \& ST. James, J. D. (1986). Visual attention within and around the field of focal attention: A zoom lens model. Perception \& Psychophysics, 40, 225-240.

ERIKSEN, C. W., \& YEH, Y. (1985). Allocation of attention in the visual field. Joumal of Experimental Psychology: Human Perception \& Performance, 11, 583-597.

Fisk, A. D. , McGee, N. D., \& Giambra, L. M. (1988). The influence of age on consistent and varied semantic-category search performance. Psychology \& Aging, 3, 323-333.

Hartley, A. A., Kieley, J. M., \& Slabach, E. H. (1990). Age differences and similarities in the effects of cues and prompts. Journal of Experimental Psychology: Human Perception \& Performance, 16, 523-538.

Hoyer, W. J., Familant, M. E. (1987). Adult age differences in the rate of processing expectancy information. Cognitive Development, 2, 59-70.

Hoyer, W. J., \& Plude, D. J. (1982). Aging and the allocation of attentional resources in visual information-processing. In $R$. Sekuler, D. Kline, \& K. Dismukes (Eds.), Aging and human visual function (pp. 245-263). New York: Alan R. Liss.

JONIDES, J. (1980). Toward a model of the mind's eye's movement Canadian Journal of Psychology, 34, 103-112.

Juola, J. F., Crouch, T., \& Cocklin, T. (1987). Voluntary control of attention near the fovea. Acta Psychologica, 64, 207-217.

LABERGE, D. (1983). The spatial extent of attention to letters and words Journal of Experimental Psychology: Human Perception \& Performance, 9, 371-379.

LABERGE, D., \& BRown, V. (1986). Variations in the size of the visual field in which targets are presented: An attentional range effect. Perception \& Psychophysics, 40, 188-200. 
LABERGE, D., \& Brown, V. (1989). Theory and measurement of attentional operations in shape identification. Psychological Review, $\mathbf{9 6}$, 101-124.

MADDEN, D. J. (1982). Age differences and similarities in the improvement of controlled search. Experimental Aging Research, 8, 91-98.

MADDEN, D. J. (1983). Aging and distraction by highly familiar stimuli during visual search. Developmental Psychology, 19, 499-507.

Madden, D. J. (1984). Data-driven and memory-driven selective attention in visual search. Journal of Gerontology, 39, 72-78.

Nissen, M. J., \& CoRKIN, S. (1985). Effectiveness of attentional cueing in older and younger adults. Journal of Gerontology, 40, 185-191.

Plude, D. J., \& Doussard-Roosevelt, J. A. (1989). Aging, selective attention, and feature integration. Psychology \& Aging, 4, 98-105.

Plude, D. J., \& Hoyer, W. J. (1986). Age and the selectivity of visual information processing. Psychology \& Aging, 1, 4-10.

Plude, D. J., Kaye, D. B., Hoyer, W. J., Post, T. A., SAynisch, M. J., \& HAHN, M. V. (1983). Aging and visual search under consistent and varied mapping. Developmental Psychology, 19, 508-512.

PosNer, M. I. (1978). Chronometric explorations of mind. Hillsdale, NJ: Erlbaum.
Posner, M. I. (1980). Orienting of attention. Quarterly Journal of Experimental Psychology, 32, 3-25.

Remington, R., \& Pierce, L. (1984). Moving attention: Evidence for time-invariant shifts of visual selective attention. Perception \& Psychophysics, 35, 393-399.

Shulman, G. L., Remington, R. W., \& McLean, J. P. (1979). Moving attention through visual space. Journal of Experimental Psychology: Human Perception \& Performance, 5, 522-526.

Shulman, G. L., Wilson, J., \& Sheehy, J. B. (1985). Spatial determinants of the distribution of attention. Perception \& Psychophysics, $37,59-65$.

TsaL, Y. (1983). Movements of attention across the visual field. Journal of Experimental Psychology: Human Perception \& Performance, 9, 523-530.

(Manuscript received August 31, 1990; revision accepted for publication January 28,1992 .) 